\title{
GESTACIÓN CUÁDRUPLE: REPORTE DE CASO Y REVISIÓN DE LA LITERATURA
}

\section{Quadruplet pregnancy: case report and review of the literature}

\author{
Alejandra Oliveros-Vargas ${ }^{1}$; Juan Carlos Otero-Pinto, $M^{2}$
}

Recibido: abril 12/16 - Aceptado: mayo 30/17

\section{RESUMEN}

Objetivo: reportar un caso de embarazo cuádruple y revisar la literatura disponible con respecto a la edad gestacional en la que se identifica el tipo de placenta (mono o policoriónica) en la que finaliza el embarazo, el peso del recién nacido y la frecuencia del cuidado prenatal de este tipo de gestaciones.

Materiales y métodos: se presenta del caso de una mujer con embarazo cuádruple secundario a técnica de reproducción asistida, atendida en una institución de cuarto nivel de complejidad, ubicada en la ciudad de Bucaramanga, Colombia. Se realizó una revisión de la literatura publicada en las bases de datos Medline vía PubMed, Embase, SciELO y UptoDate ${ }^{\circledR}$ con los términos de búsqueda: “embarazo múltiple”, “cuádruples”, "atención prenatal” y "resultado perinatal”. La búsqueda se limitó por idioma (artículos en inglés y español).

Resultados: se recuperaron 19 referencias que cumplían con los criterios de inclusión y de exclusión. No hay información sobre la frecuencia con

1 Estudiante de medicina de último año, Universidad Industrial de Santander (UIS), Bucaramanga (Colombia).

2 Médico, Escuela de Medicina Juan N. Corpas, Bogotá (Colombia); especialista en ginecoobstetricia y en medicina materno-fetal, Universidad de Costa Rica. Docente, Universidad Industrial de Santander; Fellow en ultrasonido fetal, Hospital Karolinska; Fellow en ultrasonido fetal y biopsia de vellosidades coriónicas, Hospital Karolinska; Fellow en ecocardiografía fetal y ecografía en 3D, Texas Tech University. juan_carlos_otero@yahoo.com. la que se debe hacer el control prenatal. El tipo de placenta se pudo establecer entre las semanas $9 \mathrm{y}$ 25; la edad gestacional varió entre 26 y 34 semanas. El peso varió entre 1.076 y $1.770 \mathrm{~g}$. Los embarazos multigestación se acompañan frecuentemente de complicaciones maternas y perinatales.

Conclusiones: el diagnóstico del embarazo cuádruple puede hacerse temprano en la gestación. Frecuentemente se acompaña de prematuridad y bajo peso al nacer. Se requieren estudios que evalúen el manejo más seguro y efectivo de esta condición.

Palabras clave: embarazo múltiple, cuádruples, atención prenatal, resultado del embarazo.

\section{ABSTRACT}

Objective: To report the case of a quadruplet pregnancy and conduct the review of the available literature regarding gestational age at which the type of placenta (monochorionic or polychorionic) is identified, the gestational age at which the pregnancy comes to an end, the weight of the neonate, and the frequency of prenatal care in this type of gestation.

Materials and methods: Case report of a woman with a quadruplet pregnancy secondary to assisted reproduction technique, seen at a Level IV complexity centre in the city of Bucaramanga, Colombia. A review of the literature was conducted in Medline vía PubMed, Embase, SciELO and UptoDate ${ }^{\circledR}$ 
databases using the search terms "multiple pregnancy," "quadruplets," "prenatal care," and "perinatal outcome". The search was limited by language to articles in English and Spanish.

Results: Overall, 19 references that met the inclusion and exclusion criteria were retrieved. There is no information on the required frequency of prenatal visits. It was possible to identify the type of placenta between 9 and 25 weeks; gestational age varied between 26 and 34 weeks. Weight varied between 1,076 and 1,770 g. Multigestation pregnancies are frequently associated with maternal and perinatal complications.

Conclusions: Quadruplet pregnancies may be diagnosed early in the gestation. They are frequently associated with prematurity and low birth weight. Further studies are required in order to assess the safest and most effective way of managing this condition.

Key words: Multiple pregnancy, quadruplets, prenatal care, pregnancy outcome.

\section{INTRODUCCIÓN}

La gestación multifetal es un tipo de embarazo múltiple que se define como la presencia de dos o más fetos dentro del útero (1); la frecuencia de la gestación cuádruple es de 1 por cada 500.000 nacidos vivos en el mundo (1), y se estima que el $90 \%$ ocurre como resultado de técnicas de reproducción asistida (2). Entre otros factores que incrementan la frecuencia de este tipo de gestación, también se encuentra el uso de inductores de ovulación, la edad materna avanzada, la multiparidad, la obesidad (3) y, para algunos autores, el traumatismo leve del blastocisto durante su manipulación (4). No obstante, los embarazos múltiples también pueden ser resultado de la fecundación de dos o más óvulos (bicigotos, tricigotos, cuatricigotos), y, con una menor frecuencia, de la fecundación de un solo óvulo con su ulterior división (monocigotos). Los embarazos cuádruples, entonces, pueden originarse en uno o en cuatro óvulos (4).
Con las gestaciones de alto orden fetal existe una mayor predisposición al desarrollo de complicaciones, lo que en gran parte explica la mortalidad materna asociada, que alcanza 2,5 veces más que la observada en gestaciones únicas (4). Este tipo de embarazos también incrementa el riesgo de experimentar preeclampsia, parto pretérmino, ruptura prematura de membranas, desprendimiento prematuro de placenta, pielonefritis y hemorragia posparto (3). Las gestaciones múltiples también acarrean un aumento en los costos derivados de la atención prenatal, debido a que demandan un mayor consumo de recursos (3), cifra que puede llegar a ser hasta un $40 \%$ más de aquella destinada al cuidado de las gestaciones con feto único (3), y hasta 10 veces más cuando se incluyen los costos de atención del neonato (5).

El diagnóstico se realiza, en principio, por medio de la exploración de los antecedentes personales o familiares maternos, junto con una historia clínica completa en donde se detalle la exposición a medicamentos utilizados para el tratamiento de la pareja infértil. Por otra parte, durante el examen físico se puede documentar una altura uterina mayor a la esperada, un aumento marcado de peso materno, la presencia de múltiples focos de actividad cardiaca fetal y, finalmente, a través de las maniobras de Leopold, con el hallazgo inequívoco de la presencia de dos o más fetos (4). Por supuesto, la mejor prueba diagnóstica para confirmar la multiplicidad y establecer la corionicidad es la exploración ecográfica (4).

En lo que respecta a la vía más apropiada para finalizar la gestación, la operación cesárea es la opción predilecta; no obstante, se puede producir un parto espontáneo (1). Debido a su baja incidencia, en Colombia se han descrito pocos casos que mencionen los parámetros que se deben seguir durante el cuidado prenatal de este tipo de gestaciones, por lo que el abordaje apropiado de esta condición cobra vital relevancia para el obstetra y ginecólogo. Por tanto, el objetivo de este estudio es reportar un caso de embarazo cuádruple y revisar la literatura dis- 
ponible sobre el manejo de esta entidad en cuanto a su diagnóstico ultrasonográfico, la frecuencia del control prenatal informada y el peso al nacer, y la evolución perinatal en cuanto a complicaciones y mortalidad perinatal.

\section{REPORTE DE CASO}

Gestante de 30 años de edad, gestaciones: 1, partos : 0 , con antecedente de inseminación intrauterina por infertilidad primaria, que acudió a cuidado prenatal en la unidad materno-fetal de la clínica San Luis, institución de alto nivel de complejidad, ubicada en la ciudad de Bucaramanga, Colombia, que atiende pacientes pertenecientes al régimen contributivo, en el sistema de seguridad social en Colombia.

Al momento de su ingreso la mujer cursaba con una gestación de 7 semanas 5/7 por ultrasonografía, en donde se confirmó embarazo cuádruple y se estableció la edad gestacional. El reporte de paraclínicos para el primer trimestre estuvo dentro de los parámetros esperados. Posteriormente se realizó ecografía de semana 11 0/7 a semana 13 6/7 en donde se evidenció embarazo cuádruple cuadracoriónico cuadramniótico, con traslucencia nucal, hueso nasal, ductus venoso, doppler tricuspídeo normal y anatomía satisfactoria para cada uno de los fetos. En este control prenatal se dio inicio al tratamiento profiláctico con ácido acetil salicílico $(100 \mathrm{mg})$ y calcio $(1.200 \mathrm{mg} /$ día) por riesgo de desarrollar trastorno hipertensivo del embarazo (5).

Se estimó el riesgo de trisomía 21, 18 y 13 de cada feto, con la ayuda del programa FMF-2012 (versión 2,8), llegando a la conclusión de que los fetos tenían una baja probabilidad de padecer aneuploidías. Finalmente, a esta misma edad gestacional, el doppler de arterias uterinas mostró un índice de pulsatilidad promedio de 0,70, el cual fue interpretado como negativo, con bajo riesgo ulterior de desarrollar trastornos hipertensivos asociados al embarazo o alteraciones del crecimiento fetal.

Posteriormente, en la semana 21 de gestación se realizó la ecografía de detalle y se encontraron

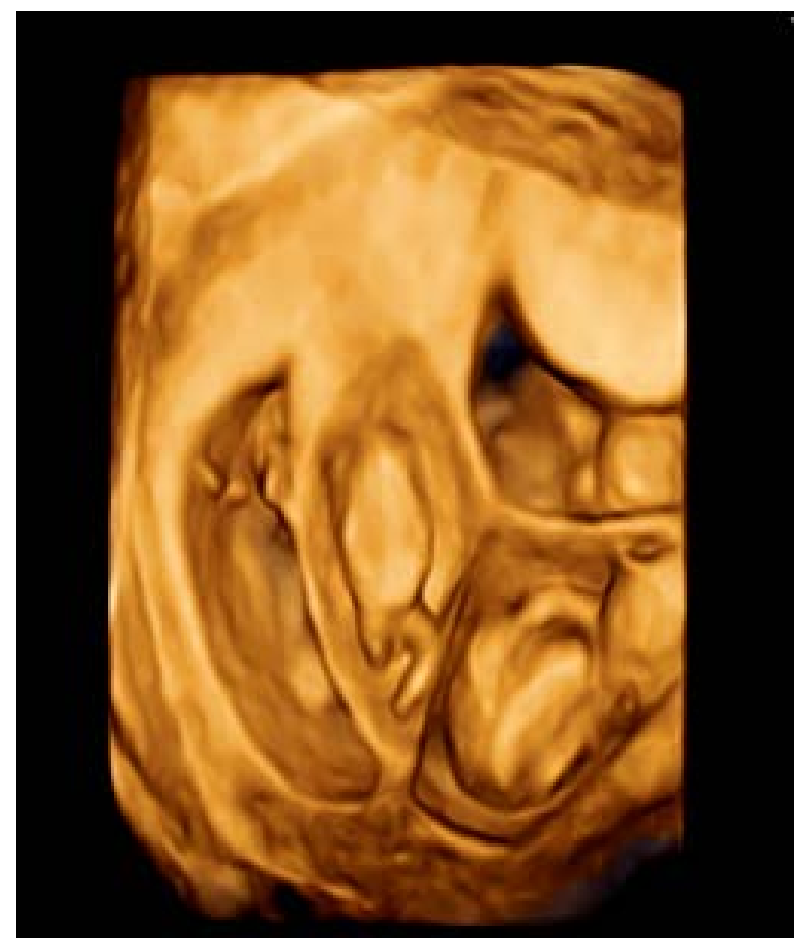

Figura 1. Ultrasonido 3D, realizado a las 21 semanas de gestación, donde se evidencian los cuatro sacos amnióticos separados, con una imagen difusa de los fetos dentro de cada saco

cuatro fetos (A, B, C y D) con un crecimiento entre percentiles 50-90, con peso fetal estimado de $400 \mathrm{a}$ $500 \mathrm{~g}$, y se evidenciaron los cuatro sacos amnióticos separados, con una imagen difusa de los fetos dentro de cada saco, como se aprecia en la figura 1. No obstante, durante el control prenatal realizado en la semana 24 de gestación se evidenció una disminución del crecimiento de los fetos $\mathrm{C}$ y D, evidente por la presencia de desaceleración en la curva de crecimiento, con pesos fetales estimados de $898 \mathrm{~g}$ con crecimiento en percentil 13,2 y 889 g con crecimiento en percentil de 12,9 respectivamente; se realizó seguimiento por medio de doppler, donde se encontró una resistencia aumentada en la arteria umbilical de un feto, por lo cual se hizo seguimiento ultrasonográfico semanal.

Se suspendió aspirina en semana 26 de gestación por la posibilidad de cesárea y posteriormente durante el control prenatal de la semana 28 se detectó la presencia de cifras tensionales estadio 
I, motivo por el cual la paciente fue hospitalizada. Durante su ingreso se documentaron cifras tensionales elevadas $(130 / 85 \mathrm{~mm} / \mathrm{Hg}, 145 / 95 \mathrm{~mm} / \mathrm{Hg}$, $148 / 90 \mathrm{~mm} / \mathrm{Hg}$ ) tomadas con intervalos de cuatro o más horas, acompañadas de cefalea intensa, por lo que se consideró que la paciente cursaba con preeclampsia; se inició esquema de sulfato de magnesio 4 g intravenoso (IV) en 30 min, y se continuó con $1 \mathrm{~g}$ IV cada hora hasta completar las 24 horas; se administraron corticoesteroides fetales, y por persistencia de cefalea, pese al manejo con sulfato de magnesio, se consideró preeclampsia severa y se decidió culminar la gestación.

La paciente se llevó a cesárea, obteniendo un primer recién nacido en posición cefálica, de sexo femenino, con un peso al nacer de $1.100 \mathrm{~g}$, talla al nacer de $37 \mathrm{~cm}$ y Apgar de 8 al minuto y de 10 a los 5 min, con cordón umbilical normal; posteriormente se realizó la extracción del segundo feto en podálica, sexo masculino, peso al nacer de $1.040 \mathrm{~g}$, talla de 38 cm y Apgar de 7 al minuto y de 10 a los 5 min, con cordón umbilical normal; luego se extrajo el tercer producto en posición podálica, de sexo femenino, peso al nacer de $985 \mathrm{~g}$, talla de $35 \mathrm{~cm}$, con Apgar de 7 al minuto y de 10 a los 5 min, con cordón umbilical normal; finalmente, se extrajo el cuarto feto en posición podálica, sexo femenino, peso al nacer de $970 \mathrm{~g}$, talla de $38 \mathrm{~cm}$, con cordón umbilical normal.

Los neonatos experimentaron síndrome de dificultad respiratoria secundario a enfermedad de membrana hialina debido a su condición de prematuridad, todos requirieron el uso de surfactante. Durante el seguimiento a mediano plazo, el recién nacido No. 1 presentó displasia broncopulmonar leve, y requirió oxígeno suplementario por 30 días; el recién nacido No. 2 desarrolló trastorno de la succión requiriendo terapia miofuncional, con una estancia hospitalaria de 41 días. El tercer fruto presentó ductus arterioso persistente que requirió tratamiento con indometacina (6), también desarrolló displasia broncopulmonar leve que requirió oxígeno suplementario por 30 días, con una estancia hospitalaria de 41 días.
Por último, el neonato número cuatro presentó sepsis tardía y enterocolitis necrotizante, la cual fue tratada quirúrgicamente y se resolvió sin complicaciones, su estancia hospitalaria fue de 48 días. Al momento de realizar este reporte, los productos de este embarazo se se encontraban en buenas condiciones y no presentaban secuelas secundarias aparentes atribuibles a la prematuridad.

En cuanto a la madre, su estancia hospitalaria fue de 10 días, se dio alta con control posterior por consulta externa, a la cual acudió 15 días después, observando buena evolución clínica. En la actualidad la paciente se encuentra en óptimas condiciones generales.

\section{MATERIALES Y MÉTODOS}

Se llevó a cabo una búsqueda de la literatura en las bases de datos Medline vía PubMed, Embase, SciELO y UptoDate ${ }^{\circledR}$, con los términos de búsqueda: "embarazo múltiple”, “cuádruples", "atención prenatal” y “resultado perinatal”. La búsqueda se limitó a los artículos publicados en inglés y español que abordaran el cuidado prenatal de los embarazos múltiples. Se incluyeron estudios tipo reporte y series de casos, metaanálisis, revisiones de tema, guías de práctica clínica y libros de consulta. Se excluyeron los estudios para los cuales no se tuvo acceso al texto completo. Se realizó una selección por título y resumen por dos autores, en caso de encontrar discrepancias se consultó con un tercer evaluador.

Los datos extraídos de los artículos seleccionados incluyeron: tipo de estudio, frecuencia de control prenatal recomendado, edad gestacional a la cual se determinó corionicidad, edad gestacional a la cual se terminó la gestación, peso fetal promedio, complicaciones perinatales.

Aspectos éticos. Se obtuvo consentimiento informado por escrito de la paciente para la publicación del caso clínico y la fotografía de los recién nacidos, y se tomaron las precauciones necesarias para garantizar la confidencialidad de la información y el anonimato de la paciente y sus hijos. 


\section{RESULTADOS}

Se recuperaron 19 referencias, las cuales en su totalidad cumplieron con los criterios de inclusión, 4 fueron reportes de caso, 6 cohortes retrospectivas, 5 revisiones de tema, 2 guías de práctica clínica, un libro de consulta y un caso control apareado. Los resultados de la revisión de los reportes de caso y los estudios retrospectivos se documentaron en la tabla 1 .
En términos globales, y basados en la literatura revisada, se puede observar que la edad gestacional a la cual se culminó el embarazo en este reporte de caso se encuentra dentro del rango observado en los casos revisados, que varió entre 26 y 35 semanas. No obstante, llama la atención que el peso al nacer promedio para los hijos de nuestra paciente (1.023 g) fue menor al promedio observado (1.046 y $1.778 \mathrm{~g})$

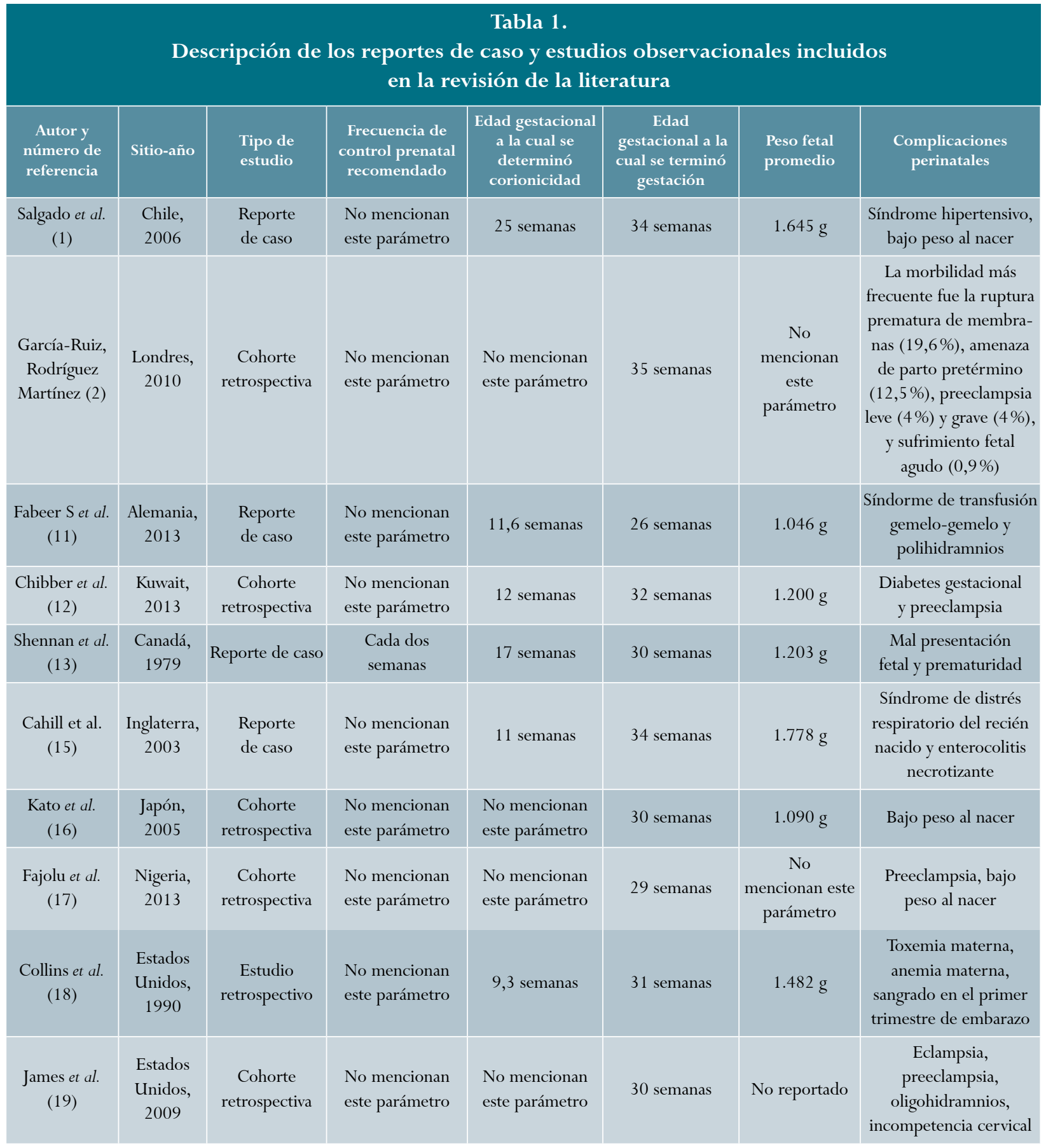


para los casos revisados. Esta diferencia podría tener su origen en la preeclampsia que presentó nuestra gestante (7), se observa además que la edad gestacional a la cual se realizó el diagnóstico de corionicidad y amniocidad en nuestra paciente estuvo cerca del límite inferior (9,3 a 25 semanas), observada en los casos revisados, ya que en nuestra paciente se realizó el diagnóstico a las 12 semanas. Los reportes de caso y estudios retrospectivos mostraron frecuentemente preeclampsia materna y complicaciones perinatales derivadas del parto pretérmino y problemas de función placentaria, por lo que surge la necesidad de un control prenatal estricto.

Al revisar la periodicidad sugerida para los controles prenatales en caso de gestación cuádruple, vale la pena recalcar que no hay información en los reportes de caso y estudios retrospectivos revisados. Sin embargo, algunos autores sugieren que su frecuencia se encuentra definida en función de la corionicidad y el número de fetos (8). El primero de los textos recuperados sugiere una frecuencia bisemanal hasta la semana 20, para luego pasar a una frecuencia semanal hasta la culminar la gestación (9). En la Guía de práctica clínica de México se recomienda que el embarazo múltiple tenga en total nueve citas de control prenatal, con frecuencia de cada dos semanas en el caso del embarazo bicorial, y en el embarazo monocorial recomiendan una vigilancia individualizada y acorde con las características de riesgo de cada mujer (3).

Aunque no hay consenso en la periodicidad en la que se deben realizar los controles prenatales en un embarazo múltiple, en la mayoría de recomendaciones se observa que se acorta el intervalo entre los controles para evitar complicaciones y para llevar el control de peso de los fetos (10); en el presente caso, el control prenatal se llevó a cabo cada dos semanas hasta la semana 20, con frecuencia semanal desde la semana 21 hasta la terminación del embarazo, lo cual contribuyó posiblemente a detectar de forma temprana el trastorno hipertensivo que presentó la paciente.
Durante el control prenatal de un embarazo múltiple se debe realizar ultrasonido semanal y describir si existe discordancia del peso fetal o del líquido amniótico (3).

Dentro de las recomendaciones para realizar los controles prenatales de un embarazo múltiple se encontró la Guía mexicana, que sugiere que estos deben ser realizados por ginecoobstetras y perinatólogos, en un centro de atención de alto nivel de

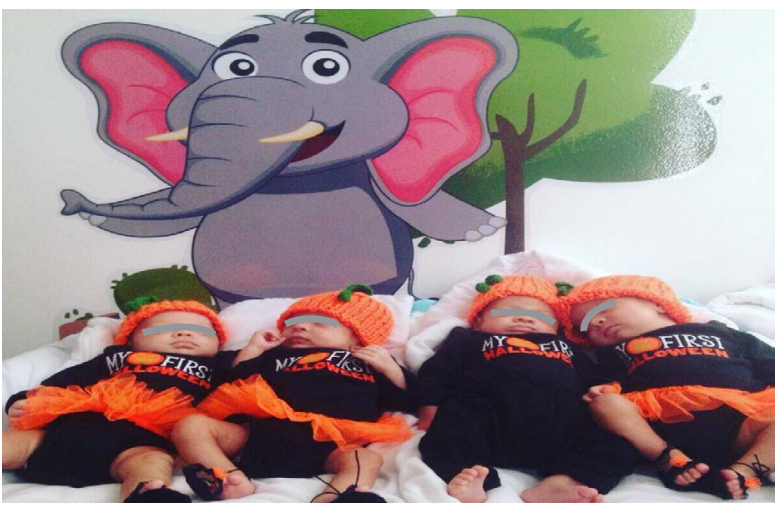

Figura 2. Cuádruples a los 5 meses

complejidad, y sugieren que se debe definir la corionicidad entre las semanas 11 y 14 de gestación; los exámenes que se deben realizar en cada trimestre son los mismos que en un embarazo único (10).

\section{CONCLUSIONES}

El manejo del embarazo cuádruple representa un reto para el obstetra a cargo del cuidado de la gestante y sus fetos. La vigilancia estrecha y frecuente del bienestar materno-fetal podría ser un punto clave a la hora de obtener un resultado favorable.

Los reportes de casos y estudios retrospectivos no brindan esta información. Se requieren estudios que describan este aspecto, así como la evolución perinatal de los recién nacidos.

\section{AGRADECIMIENTOS}

Se agradece a la paciente por brindar el consentimiento informado para publicar este caso y las fotografías que lo acompañan. 


\section{REFERENCIAS}

1. Salgado E, Lattus J. Gestación cuádruple espontánea. Un desafío para el equipo multidisciplinario en un servicio de salud. Rev Chil Obstet Ginecol. 2006 [visitado 2017 ene 20];71:35-42. Disponible en: http://www.scielo.cl/scielo.php? script $=$ sci arttext\&pid $=$ S07 17-75262006000100007\&lng $=\mathrm{e}$ $\mathrm{s} \& \mathrm{nrm}=\mathrm{iso} \& \operatorname{lng}=\mathrm{es}$

2. García-Ruiz R, Rodríguez-Martínez JA, Ortiz RME, Matute LÁ. Embarazo múltiple: incidencia, morbilidad y manejo en el centro médico ABC. An Med Asoc Med Hosp ABC. 2010 [visitado 2017 ene 20];55:122-6. Disponible en: http://www.medigraphic.com/pdfs/ abc/bc-2010/bc103c.pdf.

3. Caballero LA, Velázquez V, Segura JM, Quintanilla A, Valencia GU, García J. Guía de Práctica Clínica. Diagnóstico y manejo del embarazo múltiple. Evidencias y recomendaciones. México: Secretaría de Salud; 2013 [visitado 2017 ene 20]. Disponible en: http://www.cenetec.salud.gob.mx/ descargas/gpc/CatalogoMaestro/IMSS_628_13 EMBARAZOMULTIPLE/628GER.pdf

4. Cunninham FG. Williams Obstetricia. 23a edición. McGraw Hill interamericana; 2011. p. 859-69.

5. The American College of Obstetricians and Gynecologist ACOG. Practice advisory on low-dose aspirin and prevention of preeclampsia: updated recommendations. July 11, 2016 [visitado 2017 mar 17]. Disponible en: http://www.acog.org/About-ACOG/News-Room/ Practice-Advisories/Practice-Advisory-Low-DoseAspirin-and-Prevention-of-Preeclampsia-UpdatedRecommendations

6. Pedroza Nuñez I, Rodríguez J. Conducto arterioso: efectos de la indometacina en el feto y el recién nacido. Revista de Posgrado de la VIa Cátedra de Medicina 2002 [visitado 2017 ene 16];18-20. Disponible en: http:// med.unne.edu.ar/revista/revista122/conduc_art.html

7. Fernández Jonusas S, Ceriani Cernadas JM. Efectos de la hipertensión arterial durante el embarazo sobre el peso al nacer, el retardo del crecimiento intrauterino y la evolución neonatal. Estudio caso-control apareado. Medicina fetal y neonatología. Anales Españoles de Pediatría. 1999 [visitado 2017 mar 17];50:52-6. Disponible en: https://www.aeped.es/sites/default/ files/anales/50-1-12.pdf

8. Ramirez JV. Obstetricia Tema 0-18: embarazo múltiple. 2004 [visitado 2017 ene 21]. Disponible en: http://www.uv.es/ jvramire/apuntes/passats/ obstetricia/TEMA\%20O-18\%20(2004).pdf

9. Secretaría de Salud de México. Prevención, diagnóstico, tratamiento y referencia de la amenaza de aborto en el primer y segundo niveles de atención. Guía de referencia rápida: Guía de Práctica Clínica. México: Secretaría de Salud, CENETEC, 2015 [visitado 2017 ene 17]. Disponible en: http://www.cenetec.salud. gob.mx/descargas/gpc/CatalogoMaestro/026_GPC_ AmenazadeAborto/SSA_026_08_GRR.pdf

10. López Navarro E, Unidad de Endocrinología y Nutrición. Hospital Universitari de Girona Dr. Josep Trueta. Fisiología de la función tiroidea durante el embarazo, hipofunción tiroidea y fertilidad [visitado 2017 ene 15]. Disponible en: http://www.hvn.es/ servicios_asistenciales/ginecologia_y_obstetricia/ ficheros/actividad_docente_e_investigadora/curso de_actualizacion_en_obstetricia_y_ginecologia/ curso_2014/reproduccion/fisiologia_de_la_funcion_ tiroidea_durante_el_embarazo.pdf

11. Faber S, Ribe S, Steinke H, Thome U, Stepan H. Monochorionic quadruplet pregnancy without severe complications: Sonographic work-up and placental findings. Ultrasound Obstet Gynecol. 2013;41:707-8. doi: 10.1002/uog. 12333.

12. Chibber R, Fouda M, Shishtawy W, Al-Dossary M, Al-Hijji J, Amen A, et al. Maternal and neonatal outcome in triplet, quadruplet and quintuplet gestations following ART: A 11-year study. Arch Gynecol Obstet. 2013;288:759-67. doi: 10.1007/ s00404-013-2796-x.

13. Shennan AT, Milligan JE, Yeung PK. Successful management of quadruplet pregnancy in a perinatal unit. Can Med Assoc J. 1979;121:741-5.

14. Belaisch-Allart J. Reproducción asistida. EMC. 2011 [visitado 2015 nov 25];47:1-11. Disponible en: http://www.em-consulte.com/es/article/361236/ reproduccion-asistida.

15. Cahill DJ, Jenkins JM, Soothill PW, Whitelaw A, Wardle PG. Quadruplet pregnancy following transfer of two embryos: Case report. Hum Reprod. 2003; 18 : 441-3. doi: https://doi.org/10.1093/humrep/deg089

16. Kato N, Uchiyama Y. Reference birth-length range for multiple-birth neonates in Japan. J Obstet Gynaecol Res. 2005;31:43-9. https://doi.org/10.1111/j.14470756.2005.00248.x 
17. Fajolu IB, Ezeaka VC, Adeniyi OF, Iroha EO, EgriOkwaji MT. Prevalence and outcome of higher order multiple pregnancies in Lagos, Nigeria. J Matern Fetal Neonatal Med. 2013;26:1342-5. doi: $10.3109 / 14767058.2013 .784260$.

18. Collins MS, Bleyl JA. Seventy-one quadruplet pregnancies: Management and outcome. Am J
Obstet Gynecol. 1990;162:1384-92. https://doi. org/10.1016/0002-9378(90)90896-F

19. James S, Gil KM, Myers N, Stewart J. Effect of parity on gestacional age at delivery in multiple gestation pregnancies. J Perinatol. 2009;29:13-9. doi: 10.1038/ jp. 2008.121 .

\section{Conflicto de intereses: ninguno declarado.}

